\title{
Ultrasound-modulated optical tomography with intense acoustic bursts
}

\author{
Roger J. Zemp, Chulhong Kim, and Lihong V. Wang
}

\begin{abstract}
Ultrasound-modulated optical tomography (UOT) detects ultrasonically modulated light to spatially localize multiply scattered photons in turbid media with the ultimate goal of imaging the optical properties in living subjects. A principal challenge of the technique is weak modulated signal strength. We discuss ways to push the limits of signal enhancement with intense acoustic bursts while conforming to optical and ultrasonic safety standards. A CCD-based speckle-contrast detection scheme is used to detect acoustically modulated light by measuring changes in speckle statistics between ultrasound-on and ultrasound-off states. The CCD image capture is synchronized with the ultrasound burst pulse sequence. Transient acoustic radiation force, a consequence of bursts, is seen to produce slight signal enhancement over pure ultrasonic-modulation mechanisms for bursts and CCD exposure times of the order of milliseconds. However, acoustic radiation-force-induced shear waves are launched away from the acoustic sample volume, which degrade UOT spatial resolution. By time gating the CCD camera to capture modulated light before radiation force has an opportunity to accumulate significant tissue displacement, we reduce the effects of shear-wave image degradation, while enabling very high signal-to-noise ratios. Additionally, we maintain high-resolution images representative of optical and not mechanical contrast. Signal-to-noise levels are sufficiently high so as to enable acquisition of $2 \mathrm{D}$ images of phantoms with one acoustic burst per pixel. (c) 2007 Optical Society of America
\end{abstract}

OCIS codes: $170.3880,110.7050,110.7170,110.6150,110.6960$.

\section{Introduction}

Optical penetration and scattering limitations challenge the realization of high-resolution optical imaging of living subjects at depth scales beyond the ballistic regime. Ultrasound-modulated optical tomography (UOT) is a novel hybrid optical imaging technique that promises optical contrast with ultrasonic spatial resolution. UOT systems illuminate a specimen with a high-coherence laser source, while transmitting a focused acoustic field. Light that passes through the ultrasonic sample volume experiences modulation, which is detected in optical speckles even after multiple-scattering events. ${ }^{1}$ Modulated

When this research was performed, the authors were with the Optical Imaging Laboratory, Department of Biomedical Engineering, Texas A\&M University, College Station, Texas 77843-3120. The authors are now with the Optical Imaging Laboratory, Department of Biomedical Engineering, Washington University, One Brookings Drive, Campus Box 1097, St. Louis, Missouri 63130. L. V. Wang's e-mail address is lhwang@biomed.wustl.edu.

Received 12 June 2006; revised 4 October 2006; accepted 10 October 2006; posted 12 October 2006 (Doc. ID 71876); published 13 March 2007.

0003-6935/07/101615-09\$15.00/0

(C) 2007 Optical Society of America signals at the detector elements are assumed to originate from the ultrasonic focal volume.

Theoretical contributions explaining the mechanisms of UOT have been offered by Leutz and Maret, ${ }^{2}$ Wang, ,3,4 Sakadžić and Wang,,5,6 among others. It is now thought that two main mechanisms contribute to ultrasound-induced light modulation in optically diffuse scattering media: index of refraction variations and particle displacement resulting from the acoustic waves. A number of groups have devoted efforts to various instrumentation schemes for detection including single element detectors,${ }^{7}$ multiple parallel detectors such as CCD cameras, ${ }^{8-10}$ Fabry-Perot interferometers, ${ }^{11}$ and photorefractive crystals. ${ }^{12,13}$ Here we use a simple speckle-contrast detection scheme with a triggered CCD camera. A stochastically derived analytic explanation of the speckle-contrast detection mechanism has recently been described by Zemp et al. ${ }^{14}$

Amidst various detection techniques for measuring modulated photons, a persistent difficulty is a low signal-to-noise ratio (SNR) due to minimal acoustically induced modulation, diffuse light propagation, and uncorrelated phases among speckle grains. This paper aims to address the problem of minimal light modulation by using intense acoustic bursts. ${ }^{15}$ Most 
previous approaches in UOT have relied on $\mathrm{cw}$ or pulsed ultrasound. With a reduced duty cycle, bursts allow much greater acoustic amplitudes compared with cw ultrasound. While pulses offer superior axial resolution, the instantaneous volume of modulation is small, making detection challenging. Acoustic bursts represent a middle ground between pulses and $\mathrm{cw}$. We sacrifice axial resolution compared with pulses for the SNR, and improve upon the SNR compared with the cw case. In both situations, lateral resolution is determined by focusing the acoustic transducer.

A consequence of intense bursts is acoustic radiation force (ARF)-induced tissue motion. Micrometerscale displacements due to radiation force have been demonstrated by other groups studying ultrasonic imaging of tissue elasticity. ${ }^{16}$ In our situation, such large displacements generate greater amounts of modulated light.

Although ARF offers some signal enhancement compared with pure ultrasound-modulation mechanisms, we show that it has adverse consequences. First, if there are significant spatial variations in acoustic impedance, bulk modulus, or viscosity of tissue, ARF-weighted UOT images may be difficult to interpret as signals will be due to a complex mixture of optical and mechanical properties. Second, transient ARF generates outward-propagating shear waves that are a significant source of nonlocal optical modulation. We demonstrate that shear waves blur UOT spatial resolution and hence degrade UOT image contrast.

One way of maintaining both optical (rather than mechanical) contrast and ultrasonic spatial resolution is to acquire UOT signals near the end of a several millisecond long acoustic burst, when ARF-induced transients have attenuated sufficiently. This possibility has been discussed recently by our group. ${ }^{17}$

We demonstrate that brief CCD acquisition times synchronized with submillisecond acoustic bursts can reduce ARF-induced signal dependence, while minimizing shear-wave amplitudes. Moreover, CCD acquisition times are sufficiently brief so as to capture modulated light on time scales where shear waves are sufficiently spatially confined.

With a better understanding of signal enhancement strategies, UOT may provide greater imaging speeds, robustness to environmental perturbations, and eventually opportunities for in vivo imaging. As an illustration of the signal enhancement potential of this paper, we demonstrate high contrast-to-noise UOT images of an optical phantom without the need for signal averaging.

\section{Theory}

\section{A. Speckle-Contrast Signal Dependence on Pressure}

In Zemp et al. ${ }^{14}$ a stochastic explanation of specklecontrast detection was given. Speckle contrast is defined as

$$
C=\frac{\sigma}{\left\langle\bar{I}_{s}\right\rangle_{p}},
$$

where $\sigma$ is the standard deviation of the optical speckle pattern, averaged over all the CCD pixels $p$ and $\left\langle\bar{I}_{s}\right\rangle_{p}$ is the mean speckle intensity over all pixels $p$. The speckle contrast is related to the temporal field autocorrelation functions, described by photon path probabilities and an ensemble average of the ultrasound-induced optical phase increments. For small accumulated modulations, it was shown that the change in speckle contrast between ultrasound-on and ultrasound-off states, $\Delta C$, is proportional to the square of acoustic pressure $P_{0}^{2}$. Although the theory breaks down for large acoustic amplitudes, it does suggest a great benefit in using high acoustic pressures. Zemp et al. ${ }^{14}$ also demonstrated that lower acoustic frequencies give substantially more modulation than higher frequencies. Here we explore the use of focused $1 \mathrm{MHz}$ ultrasonic bursts to demonstrate very high SNR UOT detection.

\section{B. Radiation Force and Shear Waves: Theory}

\section{Ultrasound Radiation Force}

When ultrasound is scattered, reflected, or absorbed, energy and momentum are transferred to the medium, inducing a force, termed radiation force. Consider a focused ultrasound beam interacting with an object. The radiation force along the beam direction, $F$, is proportional to the time-averaged energy density $\langle E\rangle_{t}$ of the incident wave and the projected area of the object, $S$, as given by ${ }^{18}$

$$
F=d_{r} S\langle E\rangle_{t},
$$

where $d_{r}$, the drag coefficient, is a function of the scattered and absorbed power by the object. For the simple case of a reflecting plane target, $d_{r}$ is proportional to the power reflection coefficient. For ultrasonic scattering media, $d_{r}$ is related to the scattering cross section. The time-averaged energy density $\langle E\rangle_{t}$ of the incident wave increases with the square of acoustic pressure.

Radiation-force-induced shear waves have been studied by a number of groups. ${ }^{19-21}$ Because shear waves can adversely affect image quality, it is important to understand the generation and propagation of radiation-force-induced shear waves in tissue. Models can help provide design guidelines for UOT systems. Specifically, these principles can help us design time-gating parameters to avoid capturing shearwave-modulated optical signals.

\section{Velocity and Attenuation of Continuous-Wave Shear Waves}

The (frequency-dependent) shear-wave velocity is given as

$$
c_{t}=\sqrt{\mu / \rho},
$$

where $\mu$ is the shear elastic modulus and $\rho$ is the mass density. Shear-wave velocity is thus greater for stiffer materials. Typical values of $c_{t}$ in soft tissues 


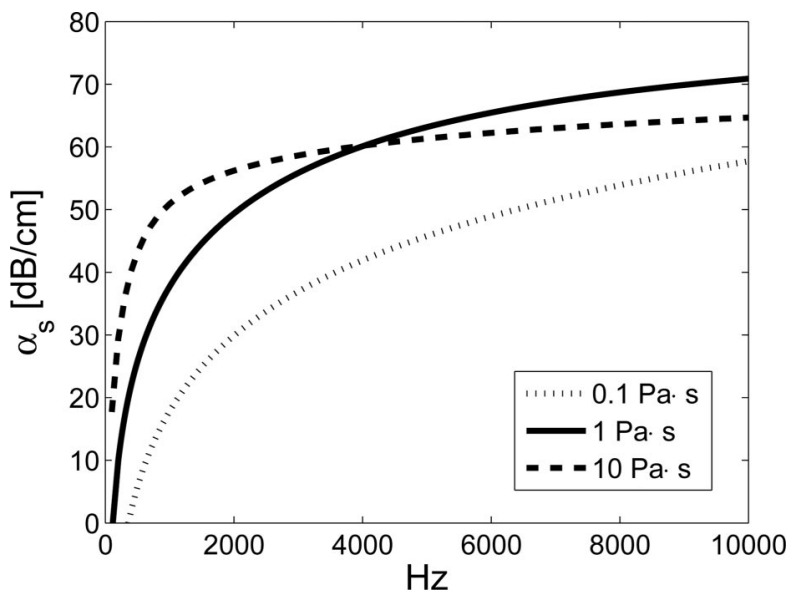

Fig. 1. Shear-wave attenuation coefficients as a function of cw shear-wave frequencies. The three curves are plotted for viscosities of $0.1,1$, and $10 \mathrm{~Pa} \mathrm{~s}$.

are of the order of a few meters per second. For CCD exposures of $0.2 \mathrm{~ms}$ trigger synchronized with ultrasonic bursts, we expect the shear wave to have traveled less than $1 \mathrm{~mm}$ during the light-integration period.

Shear-wave attenuation is also frequency dependent and largely governed by kinematic shear viscosity, given as $v=\eta / \rho$, where $\eta$ is the viscosity. In continuous oscillation regimes, the shear-wave attenuation coefficient (for plane shear waves) is determined by the real and imaginary coefficients of specific shear acoustic impedance and obtained from Frizzell et al. ${ }^{22}$ Figure 1 shows curves of attenuation coefficients with typical tissue parameters of $\mu=$ $40 \mathrm{kPa}$ and $\rho=1000 \mathrm{~kg} / \mathrm{m}^{3}$. Shear-wave attenuation is over $20 \mathrm{~dB} / \mathrm{cm}$ in the range of most physiological tissue viscosities for frequencies larger than $1 \mathrm{kHz}$.

While continuous plane-wave theory offers a rough perspective regarding shear-wave propagation velocity and attenuation, our transient radiation-force displacements are broadband and frequency dependent. Moreover, shear waves radiate outward from the pushing region hence diffractive losses will augment attenuation and improve locality. For this reason, a more complex model is appropriate.

\section{Transient Radiation Force-Induced Shear-Wave Propagation}

To gain a more complete picture of shear-wave propagation due to spatially varying and time-varying acoustic-radiation force distributions, we may use a Green's function solution for the shear-wave equation. The linear equation of shear-wave propagation is given as $^{20}$

$$
\frac{\partial^{2} s_{x}}{\partial t^{2}}-\left(c_{t}^{2}+v \frac{\partial}{\partial t}\right) \nabla_{\perp} s_{x}=F_{x}
$$

where $s_{x}$ is the medium displacement in the $x$ direction (the direction of the ultrasonic beam but perpen- dicular to the shear-wave propagation direction), $\nabla_{\perp}$ is the Laplacian operator in the transverse plane, and $F_{x}$ is the component of the ARF acting in the beam direction per unit mass.

Consider that we have a radially symmetric acoustic field that has a depth of field long enough to assume cylindrical shear-wave generation from the focal region. Then it is useful to solve the shear-wave equation in the radial frequency domain. To do so, the 2D Fourier transform in the transverse dimensions reduces to a Hankel transform so that

$$
s_{x}(r, t)=\int_{0}^{\infty} \tilde{s}(\beta, t) J_{o}(\beta r) \beta \mathrm{d} \beta,
$$

where $\beta$ is the radial spatial frequency variable conjugate to $r$, the radial distance from the origin. This allows one to write the wave equation as

$$
\frac{\mathrm{d}^{2} \tilde{s}}{\mathrm{~d} t^{2}}+\beta^{2} v \frac{\mathrm{d} \tilde{s}}{\mathrm{~d} t}+\beta^{2} c_{t}^{2} \tilde{s}=\tilde{F}_{x}(x, \beta, t) .
$$

The shear displacement $s_{x}$ can be obtained by finding the Green's function for Eq. (6) for a delta-function perturbation in time,

$$
\begin{aligned}
G\left(t, t^{\prime}\right)= & H\left(t-t^{\prime}\right) \frac{e^{-\beta^{2} v\left(t-t^{\prime}\right) / 2}}{\sqrt{\beta^{2} c_{t}^{2}-\beta^{4} v^{4} / 4}} \\
& \times \sin \left[\left(t-t^{\prime}\right) \sqrt{\beta^{2} c_{t}^{2}-\beta^{4} v^{4} / 4}\right],
\end{aligned}
$$

where $H$ is the Heaviside step function. This Green's function is a generalized version of Eq. (14) in Sarvazyan et al., ${ }^{20}$ as a delta-forcing function is assumed rather than a Gaussian form. The spatiotemporal shear-displacement profile is obtained by integrating Green's function over the radiation-force spatialfrequency profile and taking the inverse Hankel transform. Assuming that $\beta^{2} c_{t}^{2} \gg \beta^{4} v^{2} / 4$, Sarvazyan et al. provide a special-case analytic expression for the shear-displacement profile due to a focal beam profile approximated as a negative exponential form in the radial frequency domain. ${ }^{20}$ This model is sufficient to study key properties of shear-wave propagation for our problem. The shear displacement in this case takes the form of Eq. (20) in Sarvazyan et al. ${ }^{20}$

We use this model to understand cylindrical shearwave propagation in the focal plane. This model is important because shear waves impact UOT image resolution and contrast, and the model will help guide our choice of imaging parameters-especially CCD time gating. Before proceeding, we note that our model does not include nonlinear effects of shearwave propagation, which may be important for large shear amplitudes. Once a better understanding of optical modulation develops, the nonlinear effects should be included. The present model should be suf- 

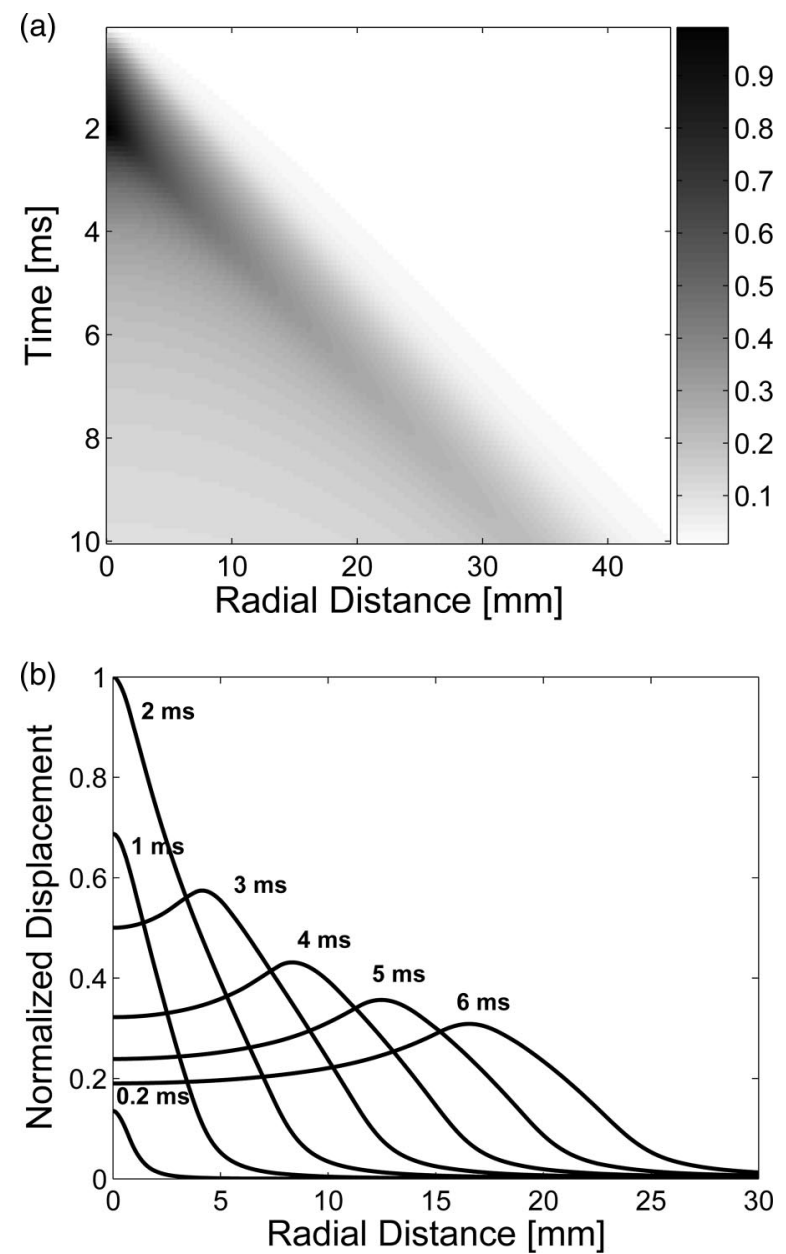

Fig. 2. (a) Shear displacement as a function of radius and time during and after a $2 \mathrm{~ms}$ acoustic burst. (b) Normalized shear displacement as a function of radial distance for various times after initiation of a $2 \mathrm{~ms}$ burst. Model parameters for both (a) and (b): Transducer aperture: $a=12.5 \mathrm{~mm}$, focal distance: $d=38 \mathrm{~mm}$, shear-wave speed: $c_{t}=4 \mathrm{~m} / \mathrm{s}$, viscosity: $\eta=0.5 \mathrm{~Pa} \mathrm{~s}$, speed of sound: $c=1500 \mathrm{~m} / \mathrm{s}$, acoustic frequency: $f_{o}=1 \mathrm{MHz}$, density: $\rho=1035 \mathrm{~kg} / \mathrm{m}^{3}$, burst duration $=2 \mathrm{~ms}$, pressure: $P_{o}=1 \mathrm{MPa}$. These parameters were selected to match conditions for experiments in Section 3.

ficient for short bursts where accumulated tissue displacements are minimal or for cases where there is reasonably weak efficiency of momentum transfer to the medium. Figure 2 shows shear-wave displacements due to a focused beam with burst duration of 2 $\mathrm{ms}$ as a function of radial position and time. Model parameters are given in the figure caption and accurately represent the parameters of our transducer and phantom properties in subsequent experiments. The shear displacement versus radius curves, shown in Fig. 2(b), are horizontal slices through Fig. 2(a). Shear displacement is seen to grow with time during the pushing phase. A steady-state distribution would develop over sufficiently long radiation-force application. The simulation results below will be discussed further within the context of experimental results in Section 3.

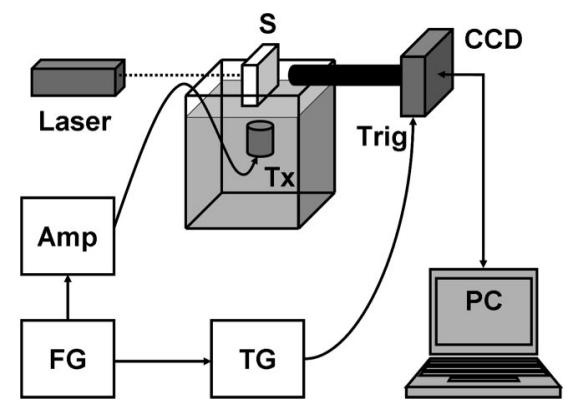

Fig. 3. Experimental setup: FG, function generator; TG, trigger generator; Tx, ultrasound transducer; $\mathrm{S}$, sample.

\section{Experiment}

Our experimental setup (Fig. 3) includes a highcoherence laser source, ultrasound transducer, imaging sample, CCD camera, and timing synchronization electronics. To acquire one point of an image, we apply ultrasonic bursts while using a CCD camera to detect modulated light originating from the ultrasonic or radiation-force sample volume. To form an image, we mechanically scan our sample relative to the light and ultrasound using a two-axis motion stage. We form images in the transverse $(x-y)$ plane relative to the $z$ (ultrasound beam) direction. We used a frequency-doubled Nd:YAG laser for its stability and long coherence length (Coherent, Verdi, $532 \mathrm{~nm}$ wavelength). For the ultrasonic field, we used a narrowband $1 \mathrm{MHz}$ transducer (Ultran VHP100-1-138) with a $25 \mathrm{~mm}$ active aperture and $38 \mathrm{~mm}$ focal length. We used $1 \mathrm{MHz}$ ultrasound because significantly more UOT signal is generated from low acoustic frequencies in comparison with high frequencies, as discussed in Ref. 14. The laser beam was aligned in free space to pass through the ultrasonic focus such that the light and ultrasound directions were perpendicular.

Speckle patterns emerging from the sample were captured by a digital CCD camera (Basler, A312f, 12 bit, 640480) so that the speckle spot sizes were matched to the CCD pixel size. A light tube with the appropriate aspect ratio was used to ensure this matching. A function generator (Agilent, 33250A) synthesized $0.2-20 \mathrm{~ms}$ bursts that were subsequently amplified by a rf power amplifier (ENI, Inc., 325LA) to drive the ultrasound transducer. Synchronization signals from the function generator triggered a trigger-delay generator (Stanford Research, DG535) that produced two CCD trigger pulses for each burst. One image was captured with the ultrasound on while the subsequent image was acquired with the ultrasound off. This method is well suited for observing transient effects and provides greater robustness to slow drifts in speckle contrast due to laser stability or environmental disturbances.

\section{A. Phantoms for Radiation-Force-Sensitive Ultrasound-Modulated Optical Tomography Imaging}

We wanted to construct phantoms with optical absorption contrast but with no mechanical or ultra- 
sonic backscatter contrast. We also wanted the phantom to possess optical scattering properties similar to human tissue, as well as ultrasonic and mechanical properties similar to tissue. We found that phantoms consisting of $10 \%$ gelatin and $10 \%$ cornstarch offered an optical reduced scattering coefficient $\mu_{s}{ }^{\prime}=9.2$ - similar to typical biological tissues. This measurement was performed using obliqueincidence reflectometry. ${ }^{23}$ The cornstarch acted as both optical and acoustic scatterers. Inclusion of ultrasonic scatterers was important to absorb acoustic momentum. Without ultrasonic scatterers, ARF has little effect above pure ultrasonic modulation.

Mechanical properties of $10 \%$ gelatin vary from sample to sample and depend on heating and cooling. Shear moduli of $10 \%$ gelatin has been measured to be within the range of $2.5-4.5 \mathrm{kPa}^{24}$ Density of the phantom is approximately $1035 \mathrm{~kg} / \mathrm{m}^{3}$, and viscosity is $0.1 \mathrm{~Pa} \mathrm{s.}{ }^{24}$ All these values are within ranges of typical biological tissues.

To create targets with optical absorption contrast, we embedded thin strips of Trypan blue-dyed phantom material into the gelatin cornstarch surrounding. The optically scattering phantom slab was further embedded in clear gelatin and encased in a four-sided acrylic box with a height of $15 \mathrm{~cm}$, length of $5 \mathrm{~cm}$, and width of $10 \mathrm{~cm}$. The box had a thin plastic membrane on the top and bottom for acoustic coupling. The casing was wide enough to allow the transducer to scan a desired region of interest without blocking the acoustic aperture.

B. Time-Gated Observation of Transient Radiation-Force and Shear-Wave Effects with Speckle-Contrast Detection

To observe transient radiation force and shear-wave effects in UOT, we designed an experiment to capture the modulated light at different CCD trigger delay times following the application of various bursts. The function generator was used to drive an ultrasound burst, while producing a trigger signal upon burst initiation. This trigger was delayed by the pulse propagation time to the focus plus $0,1,2, \ldots, 25 \mathrm{~ms}$ delays using a trigger generator. In this way, we captured different phases of the transient response of the medium. In this experiment, we used a $2 \mathrm{~cm}$ homogeneous gelatin-cornstarch phantom, $1 \mathrm{MHz}$ ultrasound transducer (1.9 MPa), and $532 \mathrm{~nm}$ laser light $(\sim 90 \mathrm{~mW})$. We varied the CCD trigger delay over a $25 \mathrm{~ms}$ period for (1) a $20 \mathrm{~ms}$ burst and (2) a 2 ms burst, using a $2 \mathrm{~ms}$ CCD exposure time-to obtain Fig. 4(a). In Fig. 4(b) we used a 0.2 ms CCD exposure time with $1 \mathrm{~W}$ of laser energy (safety limits will be discussed below) for 20 and $0.2 \mathrm{~ms}$ bursts of $1.9 \mathrm{MPa}$. We plot $\Delta C$, the change in laser speckle contrast between ultrasound-off and ultrasound-on states, as a function of the trigger-delay time. Error bars are the standard deviation of the mean of five measurements. In Fig. 4(a) an initial region of signal enhancement is seen in the first couple of milliseconds after burst initiation, then this transient effect decays to a plateau where pure ultrasound mechanisms domi-

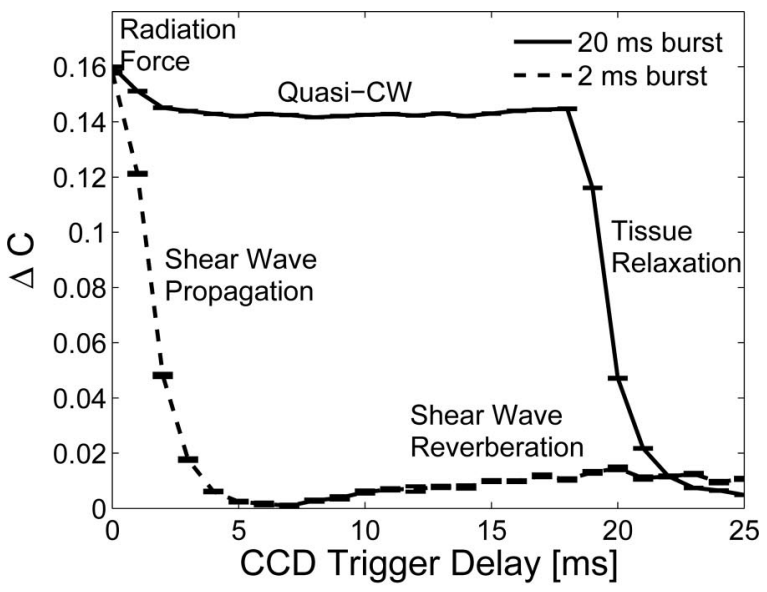

(a)

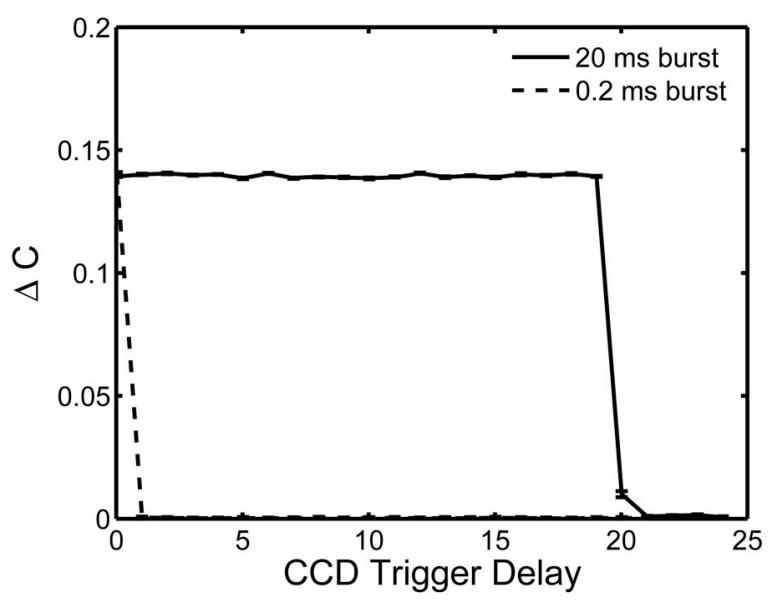

(b)

Fig. 4. Change in speckle contrast versus CCD trigger delay for (a) $2 \mathrm{~ms}$ CCD exposure time and (b) $0.2 \mathrm{~ms}$ CCD exposure time.

nate. We call this the quasi-cw regime. High SNR UOT imaging using this regime was recently discussed by our group. ${ }^{17}$

Although transient ARF offers statistically significant signal enhancement over quasi-cw methods for the same acoustic pressure, the enhancement is not as large as expected. Part of the reason may be that pure ultrasound mechanisms offer a tremendous signal.

Following the end of the $20 \mathrm{~ms}$ burst in Fig. 2(a), the change in contrast decays to near zero, however, the decay is not instantaneous. The residual decaying signal is due to tissue relaxation and subsequent shear wavefront propagation away from the pushing region and away from the region of optical illumination, as predicted by our simulations in Fig. 2. The transient effects at the beginning and end of the burst are not seen in gelatin-Intralipid phantoms absent of acoustic scatterers or absorbers (data not shown).

The effects of shear waves are more clearly seen in Fig. 2(a) in the curve for the $2 \mathrm{~ms}$ burst: specklecontrast signals are detected long after the burst. The signal decays to its minimum at $\sim 6 \mathrm{~ms}$ when shear wavefronts have propagated away from the ultrasound focal region. The shear waves reflect off the 
walls of the acrylic box encasing our phantom and are again detected as evidenced by the signal increase during 10-20 ms trigger delay times. These observation times correspond to the two-way transit times of a shear wave of a few meters per second velocity. This also explains slight perturbations in the curve for the $20 \mathrm{~ms}$ burst. The shear waves became virtually undetectable at $100 \mathrm{~ms}$.

In contrast, when we use a $0.2 \mathrm{~ms}$ CCD exposure time, shear-wave effects are much less significant. First consider the case of a $0.2 \mathrm{~ms}$ ultrasonic burst. Large ultrasound-dominated signals are detected initially, but at CCD trigger delays immediately after the burst, there are no detectable UOT signals. When $0.2 \mathrm{~ms}$ bursts are used, very little tissue displacement is generated, and importantly, any shear waves are very spatially confined as seen in Fig. 2 . The temporal shear-wave frequency generated by a $0.2 \mathrm{~ms}$ pushing pulse is $\sim 5 \mathrm{kHz}$, where shear-wave attenuation is significant (as seen in Fig. 1). There is also another reason, illustrated by the $20 \mathrm{~ms}$ burst curve in Fig. 4(b). In this case, although tissue displacement is significant, there is no significant signal enhancement due to radiation force or shear-wave mechanisms. This can be explained by considering that the differential optical scatterer displacements over the $0.2 \mathrm{~ms}$ CCD exposure time are relatively small. Short exposure times tend to filter tissue motions to reject low-frequency vibrations. This is additionally advantageous for in vivo imaging where breathing or cardiac motion is inevitable, and a good deal of robustness to such motions may be required.

\section{Loss of Spatial Resolution at High Acoustic Pressures}

Large acoustic powers induce greater acoustic and shear-wave modulation that tend to blur UOT spatial resolution and image contrast. This is seen in Fig. 5,

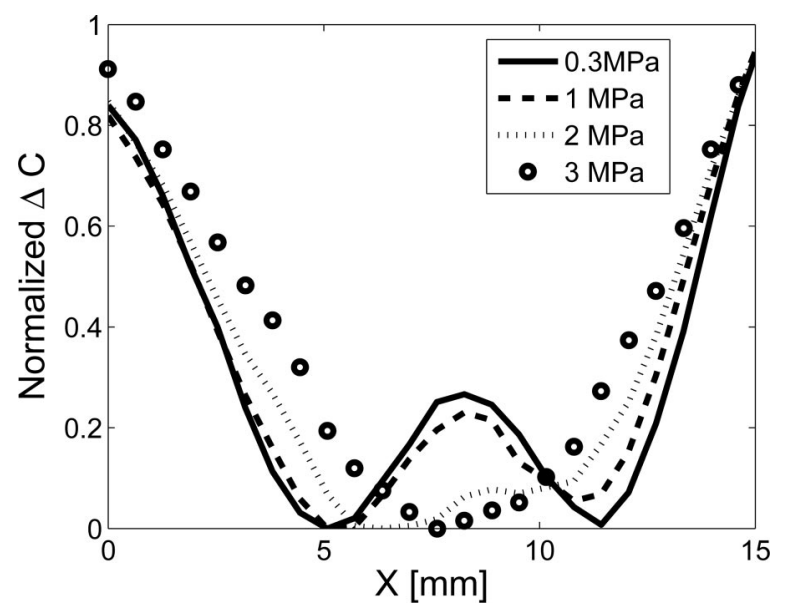

Fig. 5. Radiation-force-weighted image quality degradation as a function of increasing ultrasound pressure. The vertical axis is the measured change in speckle contrast $\Delta C$ between ultrasound-on and ultrasound-off-states, which has been shifted and normalized to map to a scale of 0 to 1 [i.e., $\left(\Delta C-\Delta C_{\min }\right) /\left(\Delta C_{\max }-\Delta C_{\min }\right)$ ]. The $x$ axis is the lateral position. The two dips representing two absorbing objects become less distinguishable for higher acoustic pressures. where $2 \mathrm{~ms}$ CCD exposure times are synchronized with the ultrasonic burst. Here $2 \mathrm{~mm} \times 2 \mathrm{~mm} \times$ $20 \mathrm{~mm}$ dark objects are separated by only $4 \mathrm{~mm}$ (6 $\mathrm{mm}$ center to center). We use close object separations to demonstrate our point. These objects are seen as dips in the signal. Object separation is visible with $1 \mathrm{MPa}$ focal pressure, and image quality is comparable with $0.3 \mathrm{MPa}$ cw excitation. However, as pressure is increased, object distinctness vanishes. This may be due to two possible effects: a larger effective ultrasonic modulation region and spatially distributed shear-wave-induced modulation. From the simulation results it is clear that the shear-wave extent can be greater than the object separation. These results show that there is an image quality cost to pushing acoustic excitations to extreme levels. A judicious choice of time gating and excitation parameters is necessary to increase the SNR without compromising spatial resolution and image contrast.

\section{Imaging Strategies}

From the simulations and experimental results, it is apparent that shear waves may produce nonlocalized light modulation, which may degrade spatial resolution and image contrast. Two types of imaging strategy can offer signal enhancement from bursts while avoiding the degrading influences of shear waves. The first strategy, discussed in Ref. 17, involves using a long burst and applying a delay before triggering CCD exposure. We refer to this strategy as quasi-cw imaging. The second technique, discussed in this paper, is to use a short intense burst and synchronize CCD triggering with the beginning of the burst, while keeping the CCD exposure time short enough to avoid shear waves propagating any great distance. We refer to this method as short-exposure burstsynchronized imaging. Shorter acoustic bursts allow a greater transducer duty cycle thus a faster imaging speed. Additionally, as will be seen, short-exposure times lead to substantially more immunity to environmentally induced speckle noise.

\section{E. Effects of Charge-Coupled Device Exposure Time on Ultrasound-Modulated Optical Tomography Spatial Resolution, Image Contrast, and Contrast to Noise}

Because shear-wave propagation endangers locality of light modulation, it is important to use time gating to reduce possible spatial resolution and contrast degradation. To illustrate this, we use short-exposure burst-synchronized imaging of a cornstarch-gelatin phantom possessing two $2 \mathrm{~mm} \times 2 \mathrm{~mm} \times 20 \mathrm{~mm}$ Trypan blue-dyed objects. One-dimensional images using 1,2 , and $4 \mathrm{~ms}$ CCD exposure times are shown in Fig. 6. In this experiment, we used 1.5 MPa pressures and burst durations matching the CCD exposure times. It is clear that the two objects, represented as dips in the signal, are most distinct for the shortest CCD exposure time.

Table 1 provides a summary of relative image contrasts and contrast-to-noise ratios (CNRs) for the various CCD exposure times. Here the CNR is defined as 


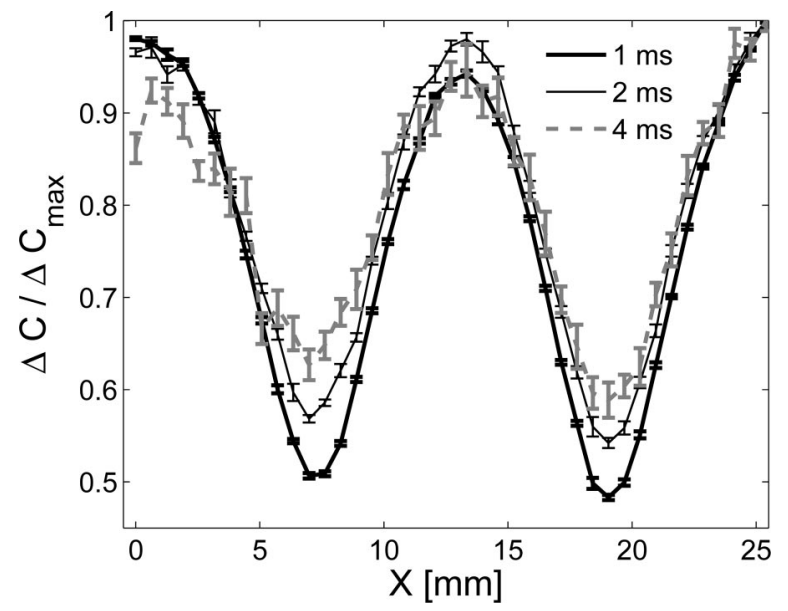

Fig. 6. Longer exposure times degrade the spatial resolution and image contrast due to integration of signal from nonlocalized shear-wave-induced displacements. Shorter-exposure times are advantageous in this regard and exhibit higher CNRs.

$$
\mathrm{CNR}=\frac{\Delta C_{\max }-\Delta C_{\min }}{\sigma_{\Delta C}},
$$

where $\Delta C_{\max }$ and $\Delta C_{\min }$ are the maximum and minimum $\Delta C$ in the image, respectively, and $\sigma_{\Delta C}$ is the standard deviation of the $\Delta C$ measurements. We interpret the improvement in the relative image contrast as a reduction of the spatial extent of the shearwave-induced signals. Additionally, it is seen that shorter-exposure times lead to significant improvement in CNR. Much of the noise in speckle-contrast measurements may be attributed to environmental vibration-induced variable speckle decorrelation. Shorter-exposure times may provide greater robustness to low-frequency vibration sources, thus the improvement in CNR.

F. High Contrast-to-Noise Ratio Images with Reasonable Spatial Resolution and Image Contrast Are Attainable Using High Pressures and Short-Exposure Times: Comparison with Quasi-cw

Here we want to compare the image quality of very high pressures and short-exposure times with the image quality due to low acoustic pressures used in early publications. ${ }^{8}$ We compare 0.3 and $1.9 \mathrm{MPa}$ bursts with CCD exposure times of $0.2 \mathrm{~ms}$. A $6 \mathrm{~ms}$ delay was introduced before capturing the $0.3 \mathrm{MPa}$ data to simulate a comparison with cw, low-intensity ultrasound. We prefer our quasi-cw approach to pure cw ultrasound as we can interlace ultrasound-on and

Table 1. Image Quality Figures of Merit as a Function of CCD Exposure Time

\begin{tabular}{ccc}
\hline $\begin{array}{c}\text { CCD Exposure Time } \\
(\mathrm{ms})\end{array}$ & Relative Contrast & CNR \\
\hline 1 & 0.52 & 169 \\
2 & 0.46 & 66 \\
4 & 0.41 & 23 \\
\hline
\end{tabular}

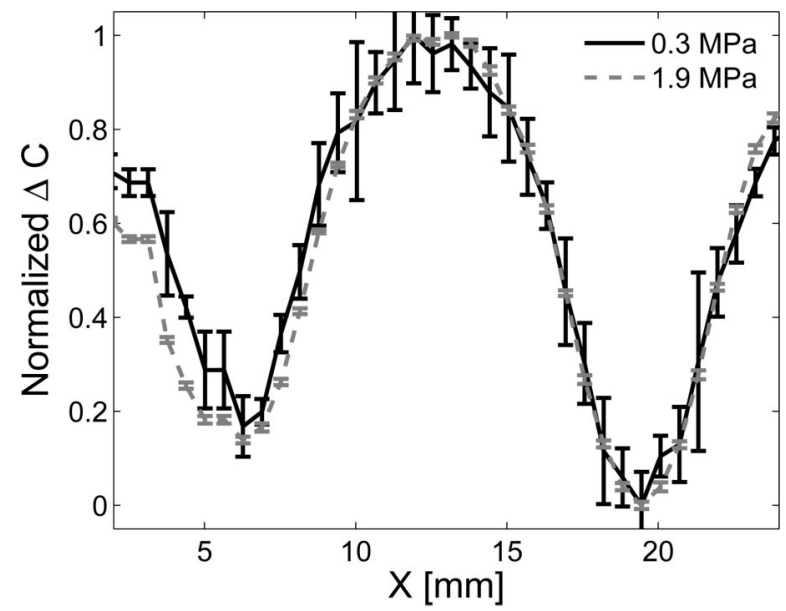

Fig. 7. Comparison of $1 \mathrm{D}$ UOT images using 0.3 and $1.9 \mathrm{MPa}$ pressures for short $0.2 \mathrm{~ms}$ CCD exposure times.

ultrasound-off acquisitions, providing greater robustness to slow quasi-random drifts in speckle contrast due to sample, laser, and environmental perturbations.

In this experiment, we use a phantom similar to previous ones, but with $1.8 \mathrm{~mm} \times 1.8 \mathrm{~mm} \times 15 \mathrm{~mm}$ and $2.4 \mathrm{~mm} \times 2.4 \mathrm{~mm} \times 15 \mathrm{~mm}$ objects. Again two distinct objects are seen as dips in Fig. 7. The vertical axis is the speckle contrast at each spatial location, mapped to a scale between zero and one.

The larger $2.5 \mathrm{~mm}$ object on the right appears to produce very similar spatial resolutions. The smaller object on the left shows a statistically significant difference between curves for 0.3 and 1.9 MPa. Even though shear waves may not be generated to any significant degree over $0.2 \mathrm{~ms}$, we may recognize that the effective region of ultrasonic modulation may be slightly larger for higher pressures than for lower pressures, and this effect is more pronounced for imaging smaller absorbing objects. This effect may also be related to a nonlinear relationship between the speckle contrast and the acoustic intensity for large acoustic pressures. Although more work is needed to better understand this phenomenon, it should be recognized that there may be a small sacrifice in spatial resolution for SNR, which is the primary objective of this paper, and a key challenge in forward progress.

Table 2 shows that the relative contrast is degraded with higher pressures, but this does not matter significantly as the CNR is very high, much larger than that due to $0.3 \mathrm{MPa}$. This is a consequence of greater absolute image contrast and low noise, in part attributable to short CCD exposure times.

Table 2. Short-Exposure-Time Image Quality Figures of Merit

\begin{tabular}{cccc}
\hline $\begin{array}{c}\text { Pressure } \\
(\mathrm{MPa})\end{array}$ & Absolute Contrast & Relative Contrast & CNR \\
\hline 1.9 & 0.053 & 0.42 & 143 \\
0.3 & 0.0096 & 0.72 & 15 \\
\hline
\end{tabular}




\section{G. Lateral Spatial Resolution}

A suitable measurement method and choice of figure of merit for spatial resolution is difficult for our imaging technique since spatial resolution and image contrast are closely related, and since we are not clear whether the image formation model is linear. For linear imaging systems, it is possible to use the pointspread function or its normalized Fourier transform (the modulation transfer function) to describe spatial resolution. Here we choose to define lateral spatial resolution as the one-way distance between the $25 \%$ and the $75 \%$ levels of an absorbing object. Similar to Kim et al. ${ }^{17}$ for pressures less than 1.5 MPa and CCD exposure times less than $2 \mathrm{~ms}$, we measure this resolution as $2.4 \pm 0.3 \mathrm{~mm}$, which is approximately equal to the acoustic beam waist at the focus.

\section{H. Two-Dimensional Imaging: No Averaging}

While previous UOT imaging techniques require averaging hundreds or thousands of times ${ }^{8,10-13}$ our signal enhancement methods allow us to significantly reduce the amount of averaging that may be required to obtain satisfactory image quality. Figure 8 shows a short-exposure burst-synchronized 2D image obtained without any averaging. The phantom is a $20 \mathrm{~mm}$ thick gelatin-cornstarch sample containing two Trypan blue-dyed objects (separated by $12 \mathrm{~mm}$, from center to center). The sizes of the two objects were approximately $2 \mathrm{~mm} \times 1.5 \mathrm{~mm} \times 15 \mathrm{~mm}$ and $2.5 \mathrm{~mm} \times$

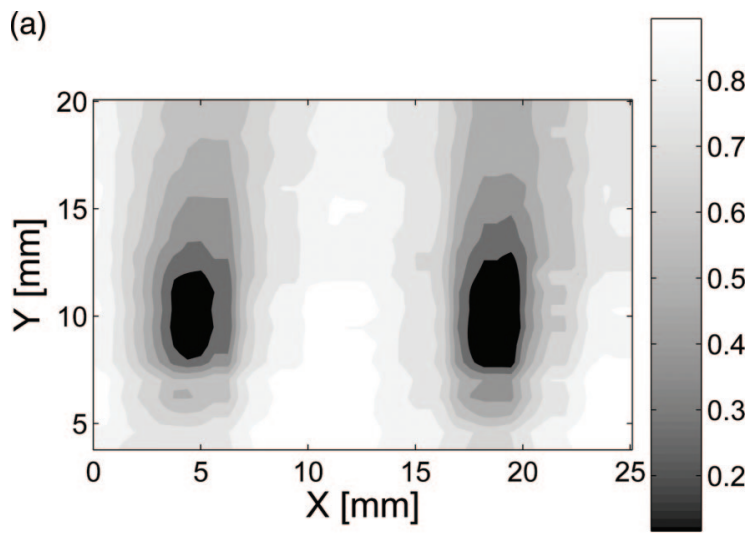

(b)

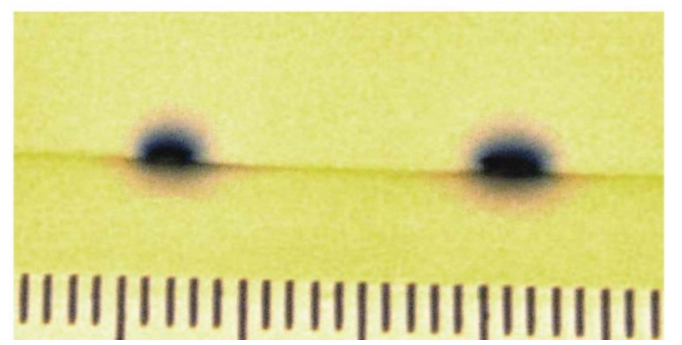

Fig. 8. (Color online) (a) Two-dimensional UOT image of a phantom with two absorbing objects to be compared with a photograph of the cross section of the phantom, shown in (b), which was cut open at the imaging plane after the UOT experiments. No averaging was used. We used intense 1.5 MPa bursts and CCD exposure time synchronized with the burst period.
$1.7 \mathrm{~mm} \times 15 \mathrm{~mm}$ along the $X, Y$, and $Z$ axes. In the image, the objects are clearly seen (Fig. 8). We used 1.5 MPa bursts of duration $0.2 \mathrm{~ms}$. The CCD camera synchronized with these bursts used an exposure time of $0.2 \mathrm{~ms}$.

\section{Discussion}

While intense bursts enable significant signal enhancement one must be aware of ARF and shear-wave effects to ensure image quality. By using shortexposure times, one avoids integrating speckle modulation due to large nonlocal shear waves generated by ARF. Short-exposure times are seen to provide greater CNR, an important consideration in moving toward animal experiments. Submillisecond exposure times may minimize interference from the hertz-kilohertz range physiological motions, but this requires greater demands on the detection sensitivity of the CCD camera or the amount of light transmitted through the tissue. Future work should also investigate optimal illumination and measurement geometries for detecting modulated light from living subjects. Other detection schemes may provide more sensitive detection, even shot-noise-limited detection of modulated light.

A discussion of safety limits is appropriate here. ANSI standards for laser safety ${ }^{25}$ permit $100 \mathrm{~mW} /$ $\mathrm{cm}^{2}$ of time-averaged $\mathrm{cw}$ laser light on the skin surface. For short-exposure times such as $0.2 \mathrm{~ms}$, we delivered $1 \mathrm{~W}$ to a phantom in a beam of $1 \mathrm{~mm}$ radius. If we were to use a shutter to illuminate the sample only during a CCD image capture time of $0.2 \mathrm{~ms}$, and we use a pulse-repetition interval of $0.8 \mathrm{~s}$ as is presently the case, the incident time-averaged laser intensity is only $4 \mathrm{~mW} / \mathrm{cm}^{2}$ if the laser beam illuminates the same position on the phantom. From this simple argument we can increase the pulserepetition rate by a factor of 25 and a good deal faster if light and ultrasound are steered to different locations from burst to burst. Additionally, even shorter pixel acquisition times may be possible with image intensifiers or reduced noise detection.

Ultrasonic safety limits should also be discussed. Mechanical index (MI), a gauge of the likelihood of cavitation, is given as $\mathrm{MI}=p_{o} / \sqrt{f_{o}}$, where $P_{o}$ is the peak ultrasonic pressure in megapascals and $f_{o}$ is the ultrasonic frequency in millihertz. For our imaging experiments using $1.5 \mathrm{MPa}$ pressure with $1 \mathrm{MHz}$ ultrasound, the MI is 1.5 , below the diagnostically acceptable limit of 1.9. In using high acoustic intensities in living subjects, we should be cautious of bioeffects, especially noting that current MI regulations are primarily for short pulses rather than long bursts. In the spirit of the American Institute of Ultrasound in Medicine (AIUM) output monitoring standard, ${ }^{26}$ one should only use a very high MI if the diagnostic information to be obtained outweighs the risk of ultrasound exposure. Tissue heating levels can be kept below biothreshold limits if burst durations are sufficiently brief. 


\section{Conclusions}

To overcome poor signal-to-noise ratios (SNRs) in ultrasound-modulated optical tomography, we have proposed the use of intense acoustic bursts to increase the amount of modulated light. From a transducer operation and ultrasound safety limit standpoint, bursts allow one to use higher peak pressures than are possible with pure cw excitation. A speckle-contrast detection scheme is used to detect the modulated signal. Experimental implementation of the specklecontrast detection method is modified such that the CCD camera exposure time is time gated to be synchronized or delayed with respect to the bursts. Synchronization offers the ability to capture transient radiation force effects that produce local displacements. These displacements generate shear waves that propagate away from the ultrasonic focus and without appropriate time gating, may be a source of loss of spatial resolution and image contrast. Submillisecond exposure times allow for the capture of a significant signal while largely avoiding the influence of shear waves. An order of magnitude CNR improvement compared with $0.3 \mathrm{MPa}$ quasi-cw ultrasound modulation was experimentally demonstrated. SNR enhancements such as discussed here may be important in moving UOT imaging technology toward the imaging of living subjects.

We thank Sava Sakadžić for fruitful discussions. We acknowledge funding from the National Institutes of Health R21/R33 CA094267.

\section{References}

1. F. A. Marks, H. W. Tomlinson, and G. W. Brooksby, "Comprehensive approach to breast cancer detection using light: photon localization by ultrasound modulation and tissue characterization by spectral discrimination," in Photon Migration and Imaging in Random Media and Tissue, B. Chance and R. R. Alfano, eds., Proc. SPIE 1888, 500-510 (1993).

2. W. Leutz and G. Maret, "Ultrasonic modulation of multiply scattered coherent light," Physica B 204, 14-19 (1995).

3. L. H. Wang, "Mechanisms of ultrasonic modulation of multiply scattered coherent light: an analytic model," Phys. Rev. Lett. 87, 043903 (2001).

4. L. H. Wang, "Mechanisms of ultrasonic modulation of multiply scattered coherent light: a Monte Carlo model," Opt. Lett. 26, 1191-1193 (2001)

5. S. Sakadžić and L. H. Wang, "Ultrasonic modulation of multiply scattered coherent light: an analytical model for anisotropically scattering media," Phys. Rev. E 66, 1-9 (2002).

6. S. Sakadžić and L. H. Wang, "Modulation of multiply scattered coherent light by ultrasonic pulses: an analytic model," Phys. Rev. E 72, 036620 (2005).

7. S. Lévêque, A. C. Boccara, M. Lebec, and H. Saint-Jalmes, "Ultrasonic tagging of photon paths in scattering media: parallel speckle modulation processing," Opt. Lett. 24, 181-183 (1999).

8. J. Li, G. Ku, and L. H. Wang, "Ultrasound-modulated optical tomography of biological tissue by use of contrast of laser speckles," Appl. Opt. 41, 6030-6035 (2002).

9. M. Kempe, M. Larionov, D. Zaslavsky, and A. Z. Genack, "Acousto-optic tomography with multiply scattered light," J. Opt. Soc. Am. A 14, 1151-1158 (1997).

10. J. Li and L. H. Wang, "Methods for parallel-detection-based ultrasound-modulated optical tomography," Appl. Opt. 41, 2079-2084 (2002).

11. S. Sakadžić and L. H. Wang, "High-resolution ultrasoundmodulated optical tomography in biological tissues," Opt. Lett. 29, 2770-2772 (2004).

12. F. Ramaz, B. C. Forget, M. Atlan, and A. C. Boccara, "Photorefractive detection of tagged photons in ultrasound modulated optical tomography of thick biological tissues," Opt. Express 12, 5469-5474 (2004).

13. T. W. Murray, L. Sui, G. Maguluri, R. A. Roy, A. Nieva, F. Blonigen, and C. A. DiMarzio, "Detection of ultrasoundmodulated photons in diffuse media using the photo-refractive effect," Opt. Lett. 29, 2509-2511 (2004).

14. R. J. Zemp, S. Sakadžić, and L. H. Wang, "Stochastic explanation of speckle contrast detection in ultrasound-modulated optical tomography," Phys. Rev. E 73, 061920 (2006).

15. A. Lev and B. G. Sfez, "Pulsed ultrasound-modulated light tomography," Opt. Lett. 28, 1549-1551 (2003).

16. K. R. Nightingale, M. S. Soo, R. W. Nightingale, and G. E. Trahey, "Acoustic radiation force impulse imaging: in vivo demonstration of clinical feasibility," Ultrasound Med. Biol. 28, 227-235 (2002).

17. C. Kim, R. J. Zemp, and L. H. Wang, "Intense acoustic bursts as a signal-enhancement mechanism in ultrasound-modulated optical tomography," Opt. Lett. 31, 2423-2425 (2006).

18. M. Fatemi and J. F. Greenleaf, "Vibro-acoustography: an imaging modality based on ultrasound-stimulated acoustic emission," Proc. Natl. Acad. Sci. USA 96, 6603-6608 (1999).

19. K. R. Nightingale, "Shear wave generation using acoustic radiation force: in vivo and ex vivo results," Ultrasound Med. Biol. 29, 1715-1723 (2003).

20. A. P. Sarvazyan, O. V. Rudenko, S. D. Swanson, B. J. Fowlkes, and S. Y. Emelianov, "Shear wave elasticity imaging: a new ultrasonic technology of medical diagnostics," Ultrasound Med. Biol. 24, 1419-1435 (1998).

21. J. Bercoff, M. Pernot, M. Tanter, and M. Fink, "Monitoring thermally-induced lesions with supersonic shear imaging," Ultrason. Imaging 26, 71-84 (2004).

22. L. A. Frizzell, E. L. Carstensen, and J. F. Dyro, "Shear properties of mammalian tissues at low megahertz frequencies," J. Acoust. Soc. Am. 60, 1409-1411 (1976).

23. G. Marquez and L. H. Wang, "White light oblique incidence reflectometer for measuring absorption and reduced scattering spectra of tissue-like turbid media," Opt. Express 1, 454-460 (1997).

24. S. Chen, "Shear property characterization of viscoelastic media using vibrations induced by ultrasound radiation force," Ph.D. dissertation (Mayo Graduate School, 2002).

25. Z136.1 ANSI Standard For the Safe Use of Lasers (American National Standards Institute, 2000).

26. AIUM/National Electrical Manufacturers Association, Standard for Real-Time Display of Thermal and Mechanical Acoustic Output Indices on Diagnostic Ultrasound Equipment (American Institute of Ultrasound in Medicine, 2004). 